\title{
Median and mode in first passage under restart
}

\author{
Sergey Belan $\odot^{*}$ \\ Department of Physics, Massachusetts Institute of Technology, Cambridge, Massachusetts 02139, USA
}

(Received 13 June 2019; accepted 24 January 2020; published 3 March 2020)

\begin{abstract}
Restart-interrupting a stochastic process followed by a new start-is known to improve the mean time to its completion, and the general conditions under which such an improvement is achieved are now well understood. Here, we explore how restart affects other important metrics of first-passage phenomena, namely, the median and the mode of the first-passage time distribution. Our analysis provides a general criterion for when restart lowers the median time and demonstrates that restarting is always helpful in reducing the mode. Additionally, we show that simple nonuniform restart strategies allow to optimize the mean and the median first-passage times, regardless of the characteristic timescales of the underlying process. These findings are illustrated with the canonical example of a diffusive search with resetting.
\end{abstract}

DOI: 10.1103/PhysRevResearch.2.013243

\section{INTRODUCTION}

The mean first-passage time is widely used to quantify performance in diverse applications ranging from randomized search algorithms to kinetics of chemical reactions [1]. Remarkably, this metrics can be significantly improved by implementation of restart, i.e., by interrupting the first-passage process just to start it anew. Speedup by restart was first noted in computer science more than two decades ago [2] with a new wave of current interest triggered by the seminal work of Evans and Majumdar [3] who demonstrated that stochastic resetting hastens diffusive search. More recently, the development of a general renewal approach has provided a unified and model-independent treatment of first passage under restart [4-6]. In particular, it has furnished a simple criterion for when restart helps to lower the expected completion time of first-passage processes and revealed universality in the behavior of the optimally restarted processes [5].

Although the mean completion time plays a central role for some applications, in many other settings, it does not capture the relevant timescale of the task, and other metrics may be more appropriate to quantify performance. More specifically, there are cases when the median time should be used instead of the mean. For example, in an enzymatic reaction with an excess of substrate molecules [see Fig. 1(b)], the time taken for a significant change in concentration of the substrate is long compared with the expected catalysis time, and thus, the latter is a more natural measure of how fast the reaction proceeds. Indeed, in the limit of large substrate concentration, the number of substrate molecules converted to products in a unit volume per second is proportional to inverse mean catalysis

\footnotetext{
*sergb27@yandex.ru

Published by the American Physical Society under the terms of the Creative Commons Attribution 4.0 International license. Further distribution of this work must maintain attribution to the author(s) and the published article's title, journal citation, and DOI.
}

time $[7,8]$. However, in the opposite case when concentration of substrate molecules is low compared to that of the enzyme molecules [Fig. 1(c)], one deals with the highly nonstationary situation characterized by fast substrate depletion [9-11], and the mean catalysis time may be not informative. The natural metric of the reaction speed in such a nonstationary situation is given by the median turnover time-the time required to convert half of the initial amount of substrate into product. Also, in the contexts of randomized search algorithms and Internet tasks, one may be interested in the typical behavior captured by the median completion time rather than in the average values which may be dominated by rare but extreme runs [2,12-14].

Here, we analyze the effect of restart on the median time of a generic first-passage process. Namely, our analysis provides a condition for when the introduction of a small restart rate reduces the median first-passage time, or more generally, a given quantile of the first-passage-time density (FPTD). Using diffusive search as an illustrative example, we show that, similar to the mean first-passage time, the median can be optimized by a careful choice of the restart rate. Since the optimal restart rate is determined by the timescale of the first-passage process, which is often unknown a priori, we also explore restart protocols whose performance is weakly sensitive to such details. In addition, we describe the effect of restart on the mode of the first-passage time distribution, i.e., on the value of the process completion time which occurs most often. It turns out that in contrast to the mean and median, which may increase or decrease in response to the introduction of restart (depending on process details), this metric cannot be increased by restart.

\section{EFFECT OF RESTART ON QUANTILES OF THE FPTD}

Consider a first-passage time process characterized by the random completion time $T$ with the FPTD $P(T)$. By definition, the $q$ th quantile is the time $T_{q}$ such that the process has the probability $0<q<1$ to finish before $T_{q}$. Say, for $q=1 / 2$, $T_{1 / 2}$ represents the median first-passage time. Clearly, this 
(a)

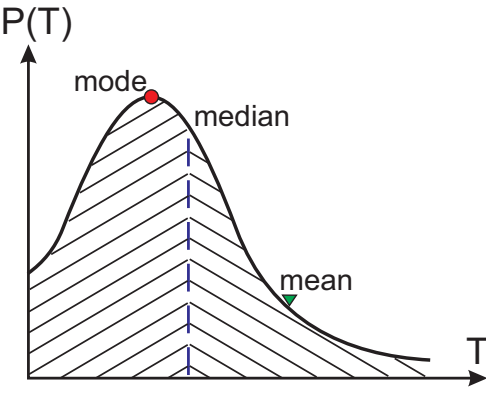

FIG. 1. (a) The mean $\langle T\rangle$, median $T_{1 / 2}$, and mode $T_{m}$ of some first-passage-time probability distribution $P(T)$. (b) Enzymatic reaction with an excess of substrate (schematically shown in blue). The reaction speed is determined by the mean turnover time. (c) Enzymatic reaction with excess enzyme (schematically shown in red); the relevant timescale of the reaction is the median turnover time.

quantity obeys the following integral equation,

$$
\int_{0}^{T_{q}} d T P(T)=q
$$

Now, let us assume that the process becomes subject to stochastic restart at the infinitesimally small rate $\delta r \rightarrow+0$. How does this affect the $q$ th quantile of this process? In the presence of restart, Eq. (1) takes the form $\int_{0}^{T_{q}+\delta T_{q}} d T P_{\delta r}(T)=$ $q$, where $\delta T_{q}$ represents the change in the $q$ th quantile and $P_{\delta r}(T)$ is the FPTD modified by the restart. It is straightforward to show that

$$
\delta T_{q} \approx-\frac{1}{P\left(T_{q}\right)} \int_{0}^{T_{q}} d T\left[P_{\delta r}(T)-P(T)\right] .
$$

To proceed, we need to know the difference $P_{\delta r}(T)-P(T)$ which determines the response of the FPTD to the introduction of rare Poisson restarts. As shown in Refs. [4,5], the Laplace transform of the FPTD $\tilde{P}_{r}(s)=\int_{0}^{\infty} d T e^{-s T} P_{r}(T)$ of any stochastic process under constant restart rate $r$ is given by

$$
\tilde{P}_{r}(s)=\frac{\tilde{P}(s+r)}{\frac{s}{s+r}+\frac{r}{s+r} \tilde{P}(s+r)} .
$$

In the limit of small restart rate $\delta r$, this equation yields

$$
\tilde{P}_{\delta r}(s) \approx \tilde{P}(s)+\left(\frac{\partial \tilde{P}(s)}{\partial s}-\frac{1}{s} \tilde{P}(s)^{2}+\frac{1}{s} \tilde{P}(s)\right) \delta r .
$$

Using the identities,

$$
\begin{gathered}
\int_{0}^{\infty} d T P(T) T e^{-s T}=-\partial_{s} \tilde{P}(s), \\
\int_{0}^{\infty} d T e^{-s T} \int_{T}^{+\infty} d t P(t)=\frac{1-\tilde{P}(s)}{s}, \\
\int_{0}^{\infty} d T e^{-s T} \int_{T}^{+\infty} d t P_{2}(t)=\frac{1-\tilde{P}^{2}(s)}{s},
\end{gathered}
$$

where $P_{2}(t) \equiv\left\langle\delta\left(T_{1}+T_{2}-t\right)\right\rangle_{T_{1}, T_{2}}=\int_{0}^{t} d \tau P(\tau) P(t-\tau)$ is the probability distribution for the sum of two independent variables $T_{1}$ and $T_{2}$ sampled from $P(T)$, we perform the inverse Laplace transform to obtain to the linear order in $\delta r$,

$$
P_{\delta r}(T)-P(T) \approx\left(\int_{T}^{\infty} d t\left[P_{2}(t)-P(t)\right]-P(T) T\right) \delta r .
$$

Next, substitution of Eq. (8) into Eq. (2) gives

$$
\delta T_{q}=-\frac{\delta r}{P\left(T_{q}\right)} \int_{0}^{T_{q}} d T\left(\int_{T}^{\infty} d t\left[P_{2}(t)-P(t)\right]-P(T) T\right) .
$$

It is easy to check that Eq. (9) gives $\delta T_{q}=0$ when $P(T)=$ $\alpha e^{-\alpha T}$. This is in accord with the general argument that restart does not affect the completion of the first-passage-time processes with exponential statistics due to their memoryless property.

From Eq. (9), we may conclude that a rigorous criterion of when restart reduces the $q$ th quantile (i.e., when $\delta T_{q}<0$ ) is provided by the inequality,

$$
\int_{0}^{T_{q}} d T\left(P(T) T+\int_{0}^{T} d t\left[P_{2}(t)-P(t)\right]\right)<0,
$$

which after integration by part becomes

$$
2\langle T\rangle_{T_{q}}-\left\langle T_{1}+T_{2}\right\rangle_{T_{q}}-T_{q} q+T_{q} K_{2}\left(T_{q}\right)<0,
$$

where $\langle T\rangle_{T_{q}}=\int_{0}^{T_{q}} d T P(T) T$ and $\left\langle T_{1}+T_{2}\right\rangle_{T_{q}}=\int_{0}^{T_{q}} d T P_{2}(T) T$ are the truncated moment, and $K_{2}(T)=\int_{0}^{T_{q}} d T P_{2}(T)$ is the cumulative distribution function of the random variable $T_{1}+$ $T_{2}$. Due to generality of the framework adopted here, this result is applicable to any first-passage process including the situations when the process is already subject to some restart mechanism.

Given the FPTD of any process of interest, one can then readily check if the inequality in Eq. (11) is fulfilled. Generally, this should be performed numerically, but the limiting cases of $q \rightarrow 0$ and $q \rightarrow 1$ allow the analytical exploration. Let us first investigate the effect of restarting on the small- $q$ quantiles. Assuming that $P(T) \approx C T^{m}$ at $T \rightarrow 0$, where $m>-1$ and $C>0$, we immediately find $\langle T\rangle_{T_{q}}=\frac{C}{m+2} T_{q}^{m+2},\left\langle T_{1}+T_{2}\right\rangle_{T_{q}}=\frac{C^{2} \Gamma^{2}(m+1)}{(2 m+3) \Gamma(2 m+2)} T_{q}^{2 m+3}, q=$ $\frac{C}{m+1} T_{q}^{m+1}, K_{2}\left(T_{q}\right)=\frac{C^{2} \Gamma^{2}(m+1)}{(2 m+2) \Gamma(2 m+2)} T_{q}^{2 m+2}, \quad$ and, therefore, Eq. (11) is satisfied only if $m<0$. Thus, restart inhibits the short-time completion of a first-passage-time process unless the FPTD $P(T)$ is singular at $T=0$. In the opposite limit, the response of the large- $q$ quantiles to restart is determined by the behavior of the tails of the FPTD. Assume that we deal with the heavy-tailed first-passage-time process having finite mean completion time $\langle T\rangle$, i.e., $P(T) \approx \frac{C}{T^{m}}$ at $T \rightarrow \infty$, where $m>2$ and $C>0$. Then, $\quad\langle T\rangle_{T_{q}}=\langle T\rangle-\frac{C}{m-2} T_{q}^{2-m},\left\langle T_{1}+T_{2}\right\rangle_{T_{q}}=2\langle T\rangle-$ $\frac{2 C}{m-2} T_{q}^{2-m}, q=1-\frac{C}{m-1} T_{q}^{1-m}, K_{2}\left(T_{q}\right)=1-\frac{2 C}{m-1} T_{q}^{1-m}$ and, thus, according to Eq. (11), restart reduces the large- $q$ quantiles of the heavy-tailed FPTD. The opposite effect is observed in the case of the fast decaying FPTD. As an example, let us consider the first-passage-time density with the Gaussian tail: $P(T) \approx C \exp \left(-\frac{T^{2}}{T_{0}^{2}}\right)$ at $T \rightarrow \infty$. Then, $\langle T\rangle_{T_{q}} \approx\langle T\rangle-\frac{C T_{0}^{2}}{2} \exp \left(-\frac{T_{q}^{2}}{T_{0}^{2}}\right),\left\langle T_{1}+T_{2}\right\rangle_{T_{q}} \approx$ $2\langle T\rangle-\sqrt{\frac{\pi}{2}} C^{2} T_{0}^{3} \exp \left(-\frac{T_{q}^{2}}{2 T_{0}^{2}}\right), q=1-\frac{\sqrt{\pi}}{2} C T_{0}\left[1-\operatorname{erf}\left(\frac{T_{q}}{T_{0}}\right)\right]$, $K_{2}\left(T_{q}\right)=1-\frac{\pi}{2} C^{2} T_{0}^{2}\left[1-\operatorname{erf}\left(\frac{T_{q}}{2 T_{0}}\right)\right]$, and Eq. (11) is not fulfilled.

It is worth noting that the above analysis is also relevant to the "deadline meeting problem" [12-15]. The probability 
that a first-passage process having the FPTD $P(T)$ will finish, before the prescribed deadline $T_{d}$ has passed, is determined by $p_{d}=\int_{0}^{T_{d}} P(T) d T$. The variational calculus based on Eq. (8) shows that, when the process is subjected to a small restart rate $\delta r$, the deadline meeting probability obtains a correction $\delta p_{d}=\left(\int_{0}^{T_{d}} d T \int_{0}^{T} d t\left[P(t)-P_{2}(t)\right]-\int_{0}^{T_{d}} d T P(T) T\right) \delta r$. Therefore, restart helps to increase the chance to meet deadline whenever $\int_{0}^{T_{d}} d T\left(P(T) T+\int_{0}^{T} d t\left[P_{2}(t)-P(t)\right]\right)<0$ or, equivalently, $2\langle T\rangle_{T_{d}}-\left\langle T_{1}+T_{2}\right\rangle_{T_{d}}-T_{d} p_{d}+T_{d} K_{2}\left(T_{d}\right)<0$.

\section{NUMERICAL RESULTS FOR ONE-DIMENSIONAI BROWNIAN SEARCH}

For the sake of illustration, let us next consider a onedimensional Brownian motion in search of an immobile (absorbing) target. In this case, the first-passage time density is given by the Levy-Smirnov distribution: $P(T)=$ $\frac{L}{\sqrt{4 \pi D T^{3}}} \exp \left(-\frac{L^{2}}{4 D T}\right)$, where $L$ is the initial distance to the target and $D$ is the diffusion coefficient. It is straightforward to verify that the $q$ th quantile of this probability density is $T_{q}=$ $\frac{L^{2}}{4 D\left[\operatorname{erfc}^{-1}(q)\right]^{2}}$. Substituting these expressions for $P(T)$ and $T_{q}$ into Eq. (10), and performing integration numerically, we find that the inequality is satisfied for $q>q_{0}$, where $q_{0} \approx 0.4123$. Thus, the introduction of restart, which occasionally returns the particle to its initial position, reduces the median completion time $T_{1 / 2}$ of diffusive search. Stochastic simulations indicate that the median time attains a minimum at the optimal restart rate $r_{0} \approx 1.5 \mathrm{D} / \mathrm{L}^{2}$ which is smaller than the rate $r^{*} \approx$ $2.5 \mathrm{D} / \mathrm{L}^{2}$ minimizing the mean search time [3]. Clearly, since restart works by avoiding the tail of the FPTD, it becomes more potent for larger $q$ as visible in Fig. 2(a). We also note in passing that restart decreases the deadline meeting probability of diffusive search for sufficiently short deadlines $T_{d}<T_{d_{0}}$ whereas increasing it for $T_{d}>T_{d_{0}}$, where $T_{d_{0}} \approx 0.7439 L^{2} / D$ [see Fig. 2(b)]. This is in accord with the analysis reported in Ref. [15] where restart is shown to increase the chance of finding a target in the presence of sufficiently small mortality rate, whereas reducing this chance if the mortality rate is large.

Clearly, to be effective, restart requires prior knowledge of the characteristic timescale of the underlying process. In the case of the median search time for diffusion, the relevant rates are measured with respect to the (inverse) diffusive timescale $\tau_{\text {dif }}=L^{2} / D$. Restart rates chosen without taking this characteristic time into account may well lead to performance that is worse than without any restart. Previously, a similar challenge motivated the development of the restart strategies improving the mean first-passage time without introducing any timescale [2,16]. The strategy proposed in Ref. [16] is make restarts separated by random scale-free time intervals. We numerically investigated the effect of such stochastic scale-free restarts on the quantiles of the diffusive search. Specifically, we implement a nonuniform restart rate which is inversely proportional to the time elapsed since the start of the process, i.e., $r(t)=\frac{\alpha}{t}$, where $\alpha$ is a dimensionless constant. Figure 3 demonstrates that such a nonuniform restart protocol allows to minimize the large- $q$ quantiles of the FPTD without being sensitive to the parameters of the problem. Indeed, the quantiles attain extrema at the optimal values $\alpha_{q}^{*}$ which do not
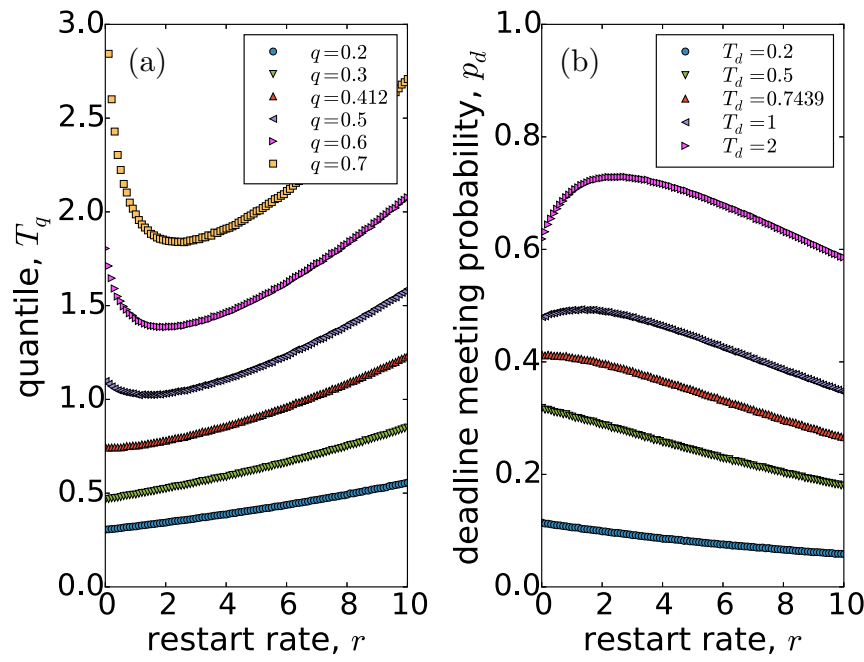

FIG. 2. (a) The $q$ th quantile $T_{q}$ of the search time distribution as a function of the rate $r$ for Poisson restarts of a one-dimensional diffusive search for an immobile target with $D=1$ and $L=1$. We see that, in agreement with theoretical predictions extracted from numerical analysis of Eq. (10), restart is helpful for $q>q_{0} \approx 0.412$. (b) The deadline meeting probability $p_{d}$ as a function of the rate $r$ of Poisson restarts for a one-dimensional diffusive search for an immobile target with $D=1$ and $L=1$. Restart is helpful for a sufficiently large time margin $T_{d}>T_{d_{0}} \approx 0.7439 L^{2} / D$. The numerical data are based on statistics including $10^{6}$ independent runs.

depend on the diffusive time $\tau_{\text {dif }}$ in contrast to the optimal rate of uniform restart which scales proportionally to $\tau_{\text {dif }}$.

We also propose and explore a nonuniform deterministic restart protocol with restart times chosen from a geometric sequence, i.e., with the $n$th restart event at $t_{n}=\tau \beta^{n-1}$, where $\tau \ll \tau_{\text {dif }}$ is a microscopic cutoff, and $\beta$ is a dimensionless
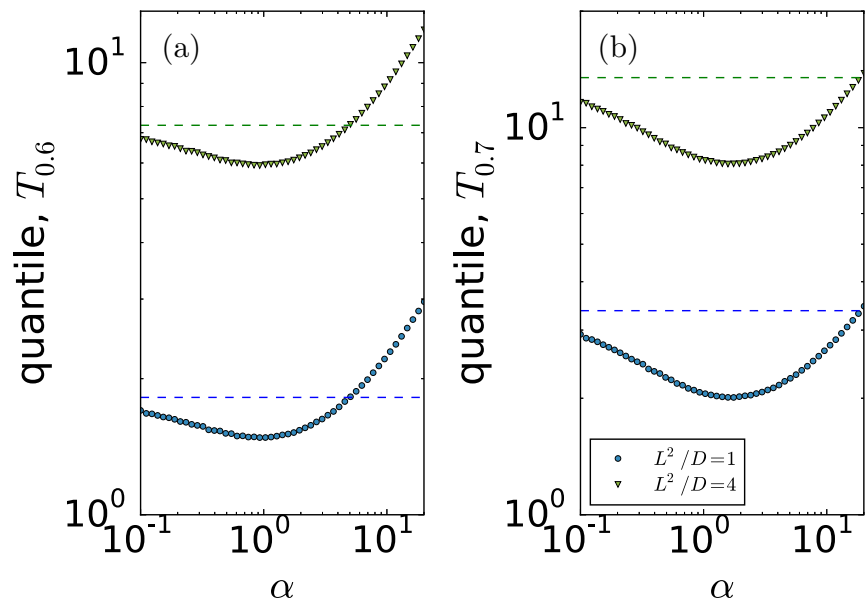

FIG. 3. Dependence of the quantiles of the search time distribution on the dimensionless parameter $\alpha$ of the stochastic scale-free restart protocol for different values of the diffusive timescale $L^{2} / D$. The dashed lines represent the unperturbed quantiles in the absence of restart. In numerical simulations, we set a short time cutoff $\tau=$ $10^{-3}$ to regularize the divergence of the restart rate at $t=0$ and collected statistics from $10^{6}$ independent realizations of the search process. 

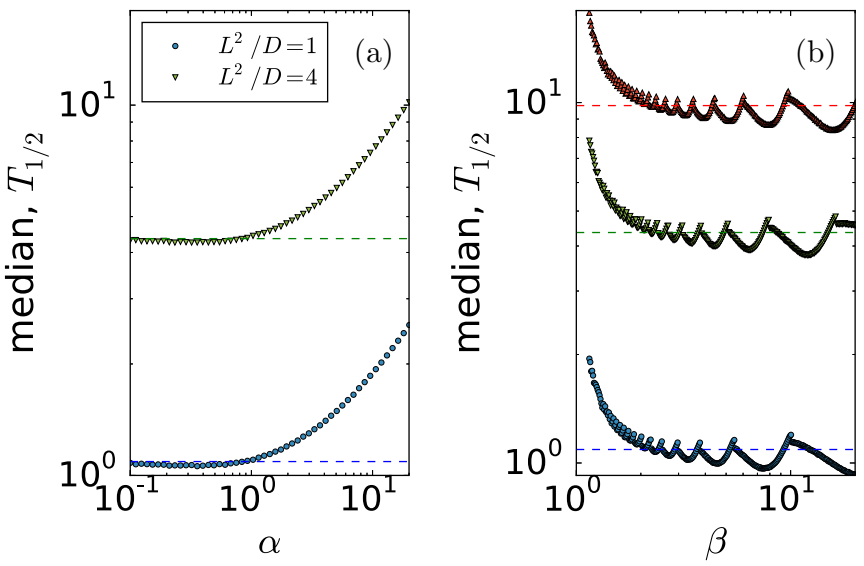

FIG. 4. The median $T_{1 / 2}$ of the search time distribution in the presence of stochastic scale-free (a), and the geometric (b) restart protocols, for different values of the diffusive timescale $L^{2} / D$. The dashed lines represent median search times in the absence of restart.

constant. In a long run, the time interval between successive restarts approaches the elapsed time since the start of the process so that there is no characteristic restart frequency. As depicted in Fig. 4(b), we find that $T_{q}$ obtains an oscillatory dependence on $\beta$, which renders practical implementation of this strategy of quantile optimization problematic. Note, however, that the geometric restart protocol is actually quite efficient for reducing the mean search time, see Fig. 5. What is more, the performance of the geometric restart in terms of the MFPT is better than that of the above-mentioned stochastic scale-free strategy. The former achieves a minimal mean search time of $\left\langle T_{\beta^{*}}\right\rangle \approx 1.8 \tau_{\text {dif }}$ at the optimal value of $\beta_{*} \approx 1.6$, that is, not sensitive to the diffusive time, whereas the latter gives $\left\langle T_{\alpha^{*}}\right\rangle \approx 1.97 \tau_{\mathrm{dif}}$ at $\alpha_{*} \approx 3.5$ [16].

\section{EFFECT OF RESTART ON THE MODE OF THE FPTD}

Finally, let us discuss another interesting metric of firstpassage processes: The mode of the FPTD, i.e., the time $T_{m}$ at which the probability distribution $P(T)$ takes its maximum
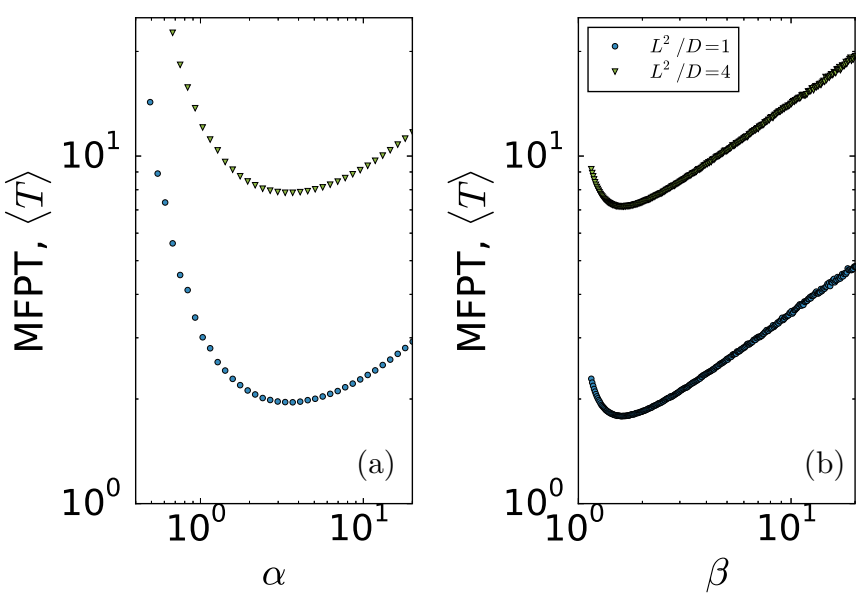

FIG. 5. The mean search time $\langle T\rangle$ in the presence of stochastic scale-free (a) and the geometric (b) restart protocols for different values of the diffusive timescale $L^{2} / D$. value. In other words, $T_{m}$ is the value of the completion time $T$ that occurs most often, which must, thus, satisfy $\left.\frac{d P(T)}{d T}\right|_{T=T_{m}}=0$, together with $\left.\frac{d^{2} P(T)}{d T^{2}}\right|_{T=T_{m}}<0$. In the presence of restarts at the small rate $\delta r$, we have $\left.\frac{d P_{\delta r}(T)}{d T}\right|_{T=T_{m}+\delta T_{m}}=0$, leading to

$$
\delta T_{m}=-\left.\frac{1}{\left.\frac{d^{2} P(T)}{d T^{2}}\right|_{T=T_{m}}} \frac{d}{d T}\left[P_{\delta r}(T)-P(T)\right]\right|_{T=T_{m}} .
$$

Next, using Eq. (8), one obtains

$$
\delta T_{m}=\frac{\delta r}{\left.\frac{d^{2} P(T)}{d T^{2}}\right|_{T=T_{m}}} \int_{0}^{T_{m}} d \tau P(\tau) P\left(T_{m}-\tau\right) .
$$

Since $\left.\frac{d^{2} P(T)}{d T^{2}}\right|_{T=T_{m}}<0$ and $\int_{0}^{T_{m}} d \tau P(\tau) P\left(T_{m}-\tau\right)=P_{2}\left(T_{m}\right) \geqslant 0$, we immediately find that $\delta T_{m} \leqslant 0$. Thus, introduction of restart decreases or leave unchanged the mode of any FPTD. Although this conclusion is based on the assumption of the infinitesimally small restart rate, the same result remains valid for any FPTD. Indeed, based on the splitting rule of the Poisson process, we can safely assume that $P(T)$ in the above formulas represents the FPTD of the process that is already subject to restart at some nonvanishing rate. Then Eq. (13) indicates that the mode of this process is a nonincreasing function of the restart rate $r$.

\section{CONCLUSION AND OUTLOOK}

In conclusion, the effectiveness of restarts in reducing the mean first-passage time has been demonstrated in a number of studies [3-6,16-32]. However, less has been known about how restart affects other characteristic time metrics of the first-passage completion. To fill this gap, we have explored the advantages of restarting to optimization of the median and mode of a generic first-passage-time density.

The ubiquity of restarts in natural and artificial systems encourages us to think that ideas presented here will find diverse applications. Say, in the context of enzymatic reactions, restarts correspond to unbinding of substrate from enzyme prior to completion of the catalytic step. Indeed, a previous analysis in the limit of large substrate concentration has shown that an increase in the substrate unbinding rate can accelerate the reaction [7]. Our results suggest that a similar effect can potentially be achieved in the opposite limit of large enzyme concentration when the reaction speed is determined by the substrate "half-life" as mentioned in the introductory part of this paper.

A range of issues call for further theoretical investigation. As proven in Refs. [2,6], deterministic (regular) restart is the universal winning strategy in the problems when one needs to minimize the mean completion time of randomized task. It remains unclear if the deterministic restart protocol is also universally optimal for the median time optimization. Another unsettled issue is if there are any rigorous bounds on the performance of the geometric restart protocol and the nonuniform scale-free stochastic restart similar to those previously obtained for the Luby's universal strategy in computer science applications [2]. 


\section{ACKNOWLEDGMENTS}

S.B. gratefully acknowledges support from the James S. McDonnell Foundation via its postdoctoral fellowship in studying complex systems. S.B. acknowledges support from NSF through Grant No. DMR-1708280. S.B. would like to thank M. Kardar for reading the paper and for providing comments.
[1] S. Redner, A Guide to First-Passage Processes (Cambridge University Press, Cambridge, UK, 2001).

[2] M. Luby, A. Sinclair, and D. Zuckerman, Optimal speedup of Las Vegas algorithms, Inf. Proc. Lett. 47, 173 (1993).

[3] M. R. Evans and S. N. Majumdar, Diffusion with Stochastic Resetting, Phys. Rev. Lett. 106, 160601 (2011).

[4] T. Rotbart, S. Reuveni, and M. Urbakh, Michaelis-Menten reaction scheme as a unified approach towards the optimal restart problem, Phys. Rev. E 92, 060101(R) (2015).

[5] S. Reuveni, Optimal Stochastic Restart Renders Fluctuations in First Passage Times Universal, Phys. Rev. Let. 116, 170601 (2016).

[6] A. Pal and S. Reuveni, First Passage under Restart, Phys. Rev. Lett. 118, 030603 (2017).

[7] S. Reuveni, M. Urbakh, and J. Klafter, Role of substrate unbinding in Michaelis-Menten enzymatic reactions, Proc. Natl. Acad. Sci. USA 111, 4391 (2014).

[8] T. Robin, S. Reuveni, and M. Urbakh, Single-molecule theory of enzymatic inhibition, Nat. Commun. 9, 779 (2018).

[9] L. A. Segel and M. Slemrod, The quasi-steady-state assumption: A case study in perturbation, SIAM Rev. 31, 446 (1989).

[10] J. A. M. Borghans, R. J. De Boer, and L. A. Segel, Extending the quasi-steady state approximation by changing variables, Bull. Math. Biol. 58, 43 (1996).

[11] S. Schnell and P. K. Maini, Enzyme kinetics at high enzyme concentration, Bull. Math. Biol. 62, 483 (2000).

[12] A. P. A. van Moorsel and K. Wolter, in MMB \& PGTS 2004, 12th GI/ITG Conference on Measuring, Modelling and Evaluation of Computer and Communiication Systems (MMB), Dresden, Germany, 2004 (VDE Verlag, Berlin, 2004), pp. 155-160.

[13] H. Wu, Randomization and restart strategies, Master's thesis, University of Waterloo, 2006.

[14] J. H. Lorenz, Completion probabilities and parallel restart strategies under an imposed deadline, PLoS ONE 11, e0164605 (2016).

[15] S. Belan, Restart Could Optimize the Probability of Success in a Bernoulli Trial, Phys. Rev. Lett. 120, 080601 (2018).

[16] Ł. Kuśmierz and T. Toyoizumi, Robust parsimonious search with scale-free stochastic resetting, Phys. Rev. E 100, 032110 (2019).

[17] M. R. Evans and S. N. Majumdar, Diffusion with optimal resetting, J. Phys. A: Math. Theor. 44, 435001 (2011).
[18] J. Whitehouse, M. R. Evans, and S. N. Majumdar, Effect of partial absorption on diffusion with resetting, Phys. Rev. E 87, 022118 (2013).

[19] M. R. Evans and S. N. Majumdar, Diffusion with resetting in arbitrary spatial dimension, J. Phys. A: Math. Theor. 47, 285001 (2014).

[20] Ł. Kuśmierz, S. N. Majumdar, S. Sabhapandit, and G. Schehr, First Order Transition for the Optimal Search Time of Lévy Flights with Resetting, Phys. Rev. Lett. 113, 220602 (2014).

[21] Ł. Kuśmierz and E. Gudowska-Nowak, Optimal first-arrival times in Lévy flights with resetting, Phys. Rev. E 92, 052127 (2015).

[22] A. Pal, A. Kundu, and M. R. Evans, Diffusion under timedependent resetting, J. Phys. A: Math. Theor. 49, 225001 (2016).

[23] S. Eule and J. J. Metzger, Non-equilibrium steady states of stochastic processes with intermittent resetting, New J. Phys. 18, 033006 (2016)

[24] A. Nagar and S. Gupta, Diffusion with stochastic resetting at power-law times, Phys. Rev. E 93, 060102(R) (2016).

[25] A. Pal, I. Eliazar, and S. Reuveni, First Passage under Restart with Branching, Phys. Rev. Lett. 122, 020602 (2019).

[26] A. Pal and V. V. Prasad, First passage under stochastic resetting in an interval, Phys. Rev. E 99, 032123 (2019).

[27] A. Masó-Puigdellosas, D. Campos, and V. Méndez, Stochastic movement subject to a reset-and-residence mechanism: Transport properties and first arrival statistics, J. Stat. Mech. (2019) 033201.

[28] L. Giuggioli, S. Gupta, and M. Chase, Comparison of two models of tethered motion, J. Phys. A: Math. Theor. 52, 075001 (2019).

[29] L. Kuśmierz and E. Gudowska-Nowak, Subdiffusive continuous-time random walks with stochastic resetting, Phys. Rev. E 99, 052116 (2019).

[30] J. Masoliver, Telegraphic processes with stochastic resetting, Phys. Rev. E 99, 012121 (2019).

[31] M. R. Evans and S. N. Majumdar, Run and tumble particle under resetting: A renewal approach, J. Phys. A: Math. Theor. 51, 475003 (2018).

[32] M. R. Evans and S. N. Majumdar, Effects of refractory period on stochastic resetting, J. Phys. A: Math. Theor. 52, 01LT01 (2018). 